\title{
TRANSFORMATIONS WITH DISCRETE SPECTRUM ARE STACKING TRANSFORMATIONS
}

\author{
ANDRÉS DEL JUNCO
}

Introduction. The stacking method (see [1] and [5, Section 6]) has been used with great success in ergodic theory to construct a wide variety of examples of ergodic transformations (see, for example, $[\mathbf{1} ; \mathbf{3} ; \mathbf{4} ; \mathbf{5} ; \mathbf{7}]$ ). However very little is known in general about the class $\mathscr{S}$ of transformations which can be constructed by the stacking method using single stacks. In particular there is no simple characterization of the class $\mathscr{S}$. In $[\mathbf{1}]$, the following question is raised: is every transformation with simple spectrum an $\mathscr{S}$-transformation? (Since the converse is true by $[\mathbf{2}$, Theorem 1], this would give a nice characterization of $\mathscr{S}$ ). The simplest case of simple spectrum is discrete spectrum and the aim of this paper is to prove that any ergodic transformation $T$ with discrete spectrum belongs to $\mathscr{S}$ (Theorem 2.3 ).

The method of proof consists in finding an increasing sequence $\left\{\mathscr{G}_{n}\right\}$ of $T$-invariant $\sigma$-algebras which generate the full $\sigma$-algebra and such that $T / \mathscr{G}_{n}$ looks like a cartesian product of several rotations and one cyclic permutation. The result is proved for this concrete case which is where the difficulty lies. One then applies a simple lemma which gives the result for $T$ itself.

I would like to thank Professor M. A. Akcoglu for suggesting this problem.

Section 0 : Notation and definitions. All measure spaces $(X, \mathscr{F}, \mu)$ will be isomorphic to the unit interval with Borel sets and Lebesgue measure. A transformation (automorphism) of $(X, \mathscr{F}, \mu)$ is an invertible bimeasurable, measure-preserving mapping of $X$ onto $X$. A partition of $X$ is a finite collection of mutually disjoint sets in $\mathscr{F}$. If $\left\{P_{n}\right\}$ is a sequence of partitions, $P_{n} \rightarrow \epsilon$ means $\mu\left(A \triangle P_{n}(A)\right) \rightarrow 0$ for all $A \in \mathscr{F}$, where $P_{n}(A)$ denotes any union of atoms of $P_{n}$ such that $\mu\left(P_{n}(A) \triangle A\right)$ is minimal. If $T$ is a transformation of $X$, a stack for $T$ (or $T$-stack) is an ordered partition $S=\left\{S_{1}, \ldots, S_{n}\right\}$ of $X$ such that $T\left(S_{j}\right)=S_{j+1}$ for $1 \leqq j<n . S_{1}$ is called the base of $S, S_{i}$ the $i$-th level and $n$ its height.

$\mathscr{S}$ is the class of transformations $T$ for which there exists a sequence $\left\{S_{n}\right\}$ of $T$-stacks such that $S_{n} \rightarrow \epsilon$ and the base of $S_{n}$ is a union of levels of $S_{n+1}$. This is just the class of transformations which can be constructed by the stacking method using single stacks. (For the stacking method see [5]). The following theorem, due to Baxter ([2, Theorem 2.1]), which we shall use implicitly, shows that the requirement that the base of $S_{n}$ be a union of levels of $S_{n+1}$ is unnecessary.

Received December 4, $197 \tilde{5}$. 
Theorem (Baxter). A transformation $T$ belongs to $\mathscr{S}$ if and only if there is a sequence $\left\{S_{n}\right\}$ of $T$-stacks such that $S_{n} \rightarrow \epsilon$.

Section 1. If $\alpha \in[0,1)$ we define the transformation $T_{\alpha}$ on $[0,1)$ by $T_{\alpha}(x)=x+\alpha(\bmod 1)$. Let $\alpha(1), \ldots, \alpha(n) \in[0,1)$ and let $\pi$ be a cyclic permutation of $S=\{1, \ldots, m\}$. Let $T=T_{\alpha(1)} \times \ldots \times T_{\alpha(m)} \times \pi$ and assume $T$ is ergodic. Denote by $(\Omega, \mathscr{F}, \mu)$ the measure space on which $T$ acts $\left(\Omega=[0,1)^{n} \times S, \mathscr{F}\right.$ the product Borel structure, $\mu$ the product of Lebesgue measures and normalized counting measure).

For each $\alpha(i)$ choose a sequence $p(i, j) / q(i, j)$ of irreducible fractions such that $q(i, j)$ increases to $\infty$ as $j \rightarrow \infty$ and

$$
\left|\alpha(i)-\frac{p(i, j)}{q(i, j)}\right| \leqq\left(\frac{1}{q(i, j)}\right)^{2} .
$$

(It is elementary and well known that this can be done. See, for example, $\left[6\right.$, Section 11.3]). Denote by $T_{j}$ the transformation

$$
T_{p(1, j) / q(1, j)} \times \ldots \times T_{p(n, j) / q(n, j)} \times \pi .
$$

Consider also the partition $Q_{i j}$ of $[0,1]$ into sets

$$
\left[\frac{r}{q(i, j)}, \frac{r+1}{q(i, j)}\right), \quad 0 \leqq r<q(i, j)
$$

and the partition $Q_{j}=Q_{i j} \times \ldots \times Q_{n j} \times \eta$ of $\Omega$ where $\eta$ denotes the partition of $S$ into points. Note that $T_{j}$ permutes the atoms of $Q_{j}$. For $\epsilon>0$ let $E_{\epsilon}=[0, \epsilon)^{n} \times\{1\} \subset \Omega$.

Lemma 1.1. Given $\epsilon>0$, there exists a $K$ such that if $x \in \Omega$ then for some $k, 0 \leqq k<K, T^{k} x \in E_{\epsilon}$.

Proof. This follows easily from the fact that the $T$-orbit of any point is dense in $\Omega$, which in turn follows easily from the ergodicity of $T$.

Lemma 1.2. Given $\epsilon>0$ there exist $K$ and $J$ such that if $j>J$ and $\xi$ is an atom of $Q_{j}$ then for some $k, 0 \leqq k<K, T_{j}{ }^{k} \xi \subset E_{\epsilon}$.

Proof. By Lemma 1.1 we can choose a $K$ such that for all $x \in \Omega$ there is a $k, 0 \leqq k<K$ such that $T^{k} x \in E_{\epsilon / 4}$. Then choose $J$ so large that

$$
K\left|\alpha(i)-\frac{p(i, j)}{q(i, J)}\right|<\frac{\epsilon}{4} \text { and } \frac{1}{q(i, J)}<\frac{\epsilon}{4}
$$

for all $i$. One checks easily that $K$ and $J$ satisfy the desired condition.

Proposition 1.3. $T \in \mathscr{S}$.

Proof. It will suffice to show that for each $\epsilon>0$ we can find a $T$-stack whose base is contained in $E_{\epsilon}$ and which covers a part of the space of measure more than $1-\epsilon$. Given $\epsilon$, then, choose $K$ and $J$ as in Lemma 1.2 and such that $2 / K<\epsilon / 2$. 
Now for any $j, Q_{j}$ breaks up into a disjoint union of $T_{j}$-stacks. Let us call these stacks $\xi(j, 1), \ldots, \xi\left(j, n_{j}\right)$. It is easy to see that they all have the same height, say $h_{j}$, and that $h_{j}$ is at least as large as $\max _{i} q(i, j)$, so that $h_{j} \rightarrow \infty$. Fix for the moment a $j$ such that $j>J, h(j)>K^{3}$ and $1 / q(i, j)<\epsilon$ for all $i$. Let $h_{j}=K^{2} D+r, 0 \leqq r<K^{2}$.

For each $n, 1 \leqq n \leqq n_{j}$, and for each $d, 1 \leqq d \leqq D$, choose a level of $\xi(j, n)$ between the $\left(d K^{2}+1\right)$-th level and the $\left(d K^{2}+K\right)$-th level which is contained in $E_{\epsilon}$ (this can be done by our choice of $J$ and $K$ ) and let $B$ be the union of all these levels. Each of these levels we have chosen has at least $K(K-1)$ images disjoint from any other chosen level, so $B$ is the base for a $T_{j}$-stack of height $K(K-1)+1$ which will cover $\Omega$ except for a set of measure less than $2 K^{2} / h(j) \leqq 2 / K<\epsilon / 2$.

We now get a $T$-stack from this $T_{j}$-stack by shrinking $B$ by a small fraction of its measure. This is done as follows. Each atom $\gamma$ of $Q_{j}$ is a product of intervals $I_{i j}^{\gamma}$ of length $1 / q(i, j), 1 \leqq i \leqq n$, and a single point in $S$. By chopping off from each end of $I_{i j}{ }^{\gamma}$ an interval of length $K(K-1)\left|\alpha_{i}-p(i, j) / q(i, j)\right|$ one gets an interval $\bar{I}_{i j}{ }^{\gamma}$ such that

$$
T_{\alpha(i)}^{l} \bar{I}_{i j}^{\gamma} \subset T_{p(i, j) / q(i, j)} I_{i j}{ }^{\gamma} \text { for } 0 \leqq l \leqq K(K-1) .
$$

(Note for future use that since $|\alpha(i)-p(i, j) / q(i, j)| \leqq(1 / q(i, j))^{2}$ we can make the amount chopped off from $I_{i j}{ }^{\gamma}$ as small a fraction of its length as we like by choosing $j$ large). It follows that if we set $\bar{\gamma}=\Pi_{i} \bar{I}_{i j}{ }^{\gamma} \times\{1\}$ then $T^{l} \bar{\gamma} \subset T_{j}^{l} \gamma$ for $0 \leqq l \leqq K(K-1)$. Finally, if $B=\cup_{\gamma \in \Gamma} \gamma$ for $\Gamma \subset Q_{j}$, we set $\bar{B}=\cup_{\gamma \in \Gamma} \bar{\gamma}$ and we have again $T^{l} \bar{B} \subset T_{j}{ }^{l} B$ for $0 \leqq l \leqq K(K-1)$. Since $\bar{I}_{i j}{ }^{\gamma}$ can be made as large a portion of $I_{i j}{ }^{\gamma}$ as we wish, the same is true of $\bar{B}$ and $B$ so that our $T$-stack can be made to cover a part of $\Omega$ of measure more than $1-\epsilon$. Of course $\bar{B} \subset B \subset E_{\epsilon}$ so this finishes the proof.

Section 2. Our aim in this section is to extend Proposition 1.3 to the case of ergodic $T$ with discrete spectrum. We begin with a simple general lemma.

Lemma 2.1. Suppose $T$ is a transformation of $(X, \mathscr{F}, \mu)$ and $\left\{\mathscr{G}_{n}\right\}$ is an incrasing sequence of $T$-invariant $\sigma$-algebras which generate. $\mathscr{F}$ such that $\left.T\right|_{\mathscr{G}_{n}} \in \mathscr{S}$. Then $T \in \mathscr{S}$.

Proof. If $\Sigma$ is a $\sigma$-algebra and $\left\{E_{n}\right\}$ is a sequence of sets in $\Sigma$ we'll say $\left\{E_{n}\right\}$ is an approximating sequence for $\Sigma$ if for each $E \in \Sigma$ and $\epsilon>0$ there is an $E_{n}$ such that $\mu\left(E_{n} \triangle E\right)<\epsilon$. Let $\left\{E_{n}\right\}$ be a sequence of sets in $\cup_{n} \mathscr{G}_{n}$ which contains an approximating sequence for each $\mathscr{G}_{n}$. Since any set in $\mathscr{F}$ can be approximated arbitrarily well by sets in $\bigcup_{n} \mathscr{G}_{n}$ it follows that $E_{n}$ is an approximating sequence for $\mathscr{F}$. Now for each $n,\left\{A_{1}, \ldots, A_{n}\right\} \subset G_{m}$ for some $m$ and since $\left.T\right|_{\mathscr{S}_{m}} \in \mathscr{S}$ we can find a ( $\mathscr{G}_{m}$-measurable) $T$-stack $S_{n}$ such that $\mu\left(A_{i} \triangle S_{n}(A)\right)<1 / n$ for $1 \leqq i \leqq n$. Then it is clear that $\mu\left(A \triangle S_{n}(A)\right) \rightarrow 0$ for every $A \in \mathscr{F}$. 
Now let $T$ be an ergodic transformation with discrete spectrum (see [8, p. 46] for the definition). Let $\left\{\lambda_{i}\right\}$ be an $\in$ numeration of the eigenvalues of the induced unitary operator and suppose $f_{i}$ is an eigenvector with eigenvalue $\lambda_{i}$. Let $\mathscr{A}_{n}$ denote the complex algebra of functions generated by $\left\{f_{i}, \bar{f}_{i}: i=1, \ldots, n\right\}$. Note that $\mathscr{A}_{n} \subset \mathscr{L}_{\infty} \subset \mathscr{L}_{2}$. Denote by $\mathscr{H}_{n}$ the $\mathscr{L}_{2}$ closure of $\mathscr{A}_{n}$. Let $\mathscr{G}_{n}$ denote the $\sigma$-algebra of sets generated by $f_{1}, \ldots, f_{n}$ (that is, the $\sigma$-algebra generated by $\left\{f_{i}^{-1}(B): i=1, \ldots, n, B\right.$ a borel set $\left.\}\right)$. Note that $\mathscr{G}_{n}$ is $T$ invariant.

Lemma 2.2. $\mathscr{H}_{n}=\mathscr{L}_{2}\left(X, \mathscr{G}_{n}, \mu\right)$.

Proof. This can be shown using the Stone-Weierstrass theorem together with some straightforward measure-theoretic arguments.

Theorem 2.3. $T \in \mathscr{S}$.

Proof. Lemma 2.2 implies that $\left.T\right|_{\mathscr{G}_{n}}$ has discrete spectrum and that its set of eigenvalues is the multiplicative group generated by $\left\{\lambda_{1}, \ldots, \lambda_{n}\right\}$. This group can be generated by a set $\left\{e^{2 \pi i \alpha(j)}: j=1, \ldots, r\right\}$ where $\{\alpha(1), \ldots, \alpha(r)\}$ is independent over the rationals. Supposing for convenience that $\alpha(r)=1 / m$ is the sole rational member of this set, we have by the discrete spectrum theorem $\left(\left[8\right.\right.$, p. 46]) that $\left.T\right|_{\mathscr{G}_{n}}$ is isomorphic to $T_{\alpha(1)} \times \ldots \times T_{\alpha(r-1)} \times \pi$ where $\pi$ is a cyclic permutation of $\{1, \ldots, m\}$. Thus $\left.T\right|_{\mathscr{G}_{n}} \in \mathscr{S}$ by Proposition 1.3. In view of Lemma 2.1 we need only show that $\mathscr{G}_{n} \uparrow \mathscr{F}$ to complete the proof. But this follows immediately from the fact that $\mathscr{L}_{2}\left(X, \mathscr{G}_{n}, \mu\right) \uparrow \mathscr{L}_{2}(X, \mathscr{F}, \mu)$.

\section{REFERENCES}

1. J. R. Baxter, A class of ergodic automorphisms, Ph.D. Thesis, University of Toronto (1969).

2. - A class of ergodic transformations having simple spectrum, Proc. Amer. Math. Soc. 24 (1970).

3. R. V. Chacon, A geometric construction of measure preserving transformations, Fifth Berkeley Symposium on Probability and Statistics (2) 2 (1967), 33;-360.

4. - Weakly mixing transformations which are not strongly mixing, Proc. Amer. Math. Soc. 22 (1969), 559-562.

5. N. A. Friedman, Introduction to ergodic theory (Van Nostrand Reinhold, 1970).

6. G. H. Hardy and E. M. Mright, An introduction to the theory of numbers, fourth edition (Oxford University Press 1960).

7. D. S. Ornstein, On the root problem in ergodic theory, Proc. 6th Berkeley Symp. Math. Stat. Prob. II (Univ. of Calif. Press 1967), 347-356.

8. P. R. Halmos, Ergodic theory (Chelsea 1956).

University of British Columbia, Vancouver, British Columbia 